Pesq. Vet. Bras. 38(5):991-996, maio 2018

\title{
Colesterol carregado pela ciclodextrina sobre a criopreservação dos espermatozoides de garanhões da raça Nordestina ${ }^{1}$
}

\author{
Wildelfrancys L. Souza ${ }^{2 *}$, Elenice A. Moraes ${ }^{3}$ e Raimundo P. Oliveira ${ }^{4}$
}

\begin{abstract}
Souza W.L., Moraes E.A. \& Oliveira R.P. 2018. [Cholesterol-loaded cyclodextrin on the cryopreservation of stallion sperm of the Northeastern breed.] Colesterol carregado pela ciclodextrina sobre a criopreservação dos espermatozoides de garanhões da raça Nordestina. Pesquisa Veterinária Brasileira 38(5):991-996. Programa de Pós-Graduação em Ciências Veterinárias, Universidade Estadual do Ceará, Avenida Dr. Silas Muguba 1700, Campus do Itaperi, Fortaleza, CE 60740-000, Brazil. E-mail: wilde@zootecnista.com.br

In the study effect of cholesterol was evaluated on the sperm quality of Nordestina stallion breed. Twenty semen samples were used from two stallions, diluted with BotuSemen extender and cholesterol add as follows: control, $0.75 \mathrm{mg}$ of cholesterol-loaded cyclodextrin (CLC) and $1.0 \mathrm{mg}$ of CLC $/ 120 \times 10^{6} \mathrm{sperm} / \mathrm{mL}$, and incubated for $15 \mathrm{~min}$ at $22^{\circ} \mathrm{C}$. The samples were diluted 1:5 with lactose-yolk egg extender and cooled to $5^{\circ} \mathrm{C}$ over two hours, loaded into $0.5 \mathrm{~mL}$ straws and frozen in static liquid nitrogen vapor before being plunged into nitrogen. Samples were thawed $\left(37^{\circ} \mathrm{C} / 30 \mathrm{~s}\right)$ and analyzed. The variables were analyzed by ANOVA and means compared by Tukey test $(\mathrm{P}<0.05)$. Higher percentages of total and progressive motile was higher for sperm treated with CLC compared to control, and CLC promoted higher percentages $(\mathrm{P}<0.05)$ of total and progressive motility for the 3 hours of incubation. The percentage of viability and plasma membrane integrity of spermatozoa were higher $(\mathrm{P}<0.05)$ in sperm treated with CLC compared to the control group. The number of spermatozoa reacted to hypoosmotic test was higher $(\mathrm{P}<0.05)$ in sperm treated with CLC than control. Addition of CLC in the semen of Nordestina stallion breed improve the sperm quality after cryopreservation.
\end{abstract}

INDEX TERMS: Cholesterol, cyclodextrin, cryopreservation, stallation, sperm, horses, Equus caballus, longevity, motility, plasma membrane, viability.

RESUMO.- Nesta pesquisa avaliou-se o efeito do colesterol sobre o sêmen de garanhões da raça Nordestina sobre a qualidade espermática. Vinte ejaculados de dois garanhões foram diluídos com BotuSemen e colesterol carreado pela ciclodextrina (CCC) adicionado no sêmen: controle, $0,75 \mathrm{mg}$ de CCC e $1,0 \mathrm{mg}$ de CCC $/ 120 \times 10^{6} \mathrm{sptz} / \mathrm{mL}$, e incubado a $26^{\circ} \mathrm{C} / 15 \mathrm{~min}$. O sêmen foi diluído 1:5 (v/v) com diluente

\footnotetext{
${ }^{1}$ Recebido em 18 de maio de 2017.

Aceito para publicação em 30 de maio de 2017.

2 Programa de Pós-Graduação em Ciências Veterinárias, Universidade Estadual do Ceará (UECE), Avenida Dr. Silas Muguba 1700, Campus do Itaperi, Fortaleza, CE 60740-000, Brasil. *Autor para correspondência: wilde@zootecnista.com.br

${ }^{3}$ Programa de Pós-Graduação em Ciência Animal, Universidade Federal do Vale do São Francisco (Univasf), Rodovia 407 Km 12, Lote 543, Projeto Nilo Coelho C1, Petrolina, PE 56300-000, Brasil.E-mail: elenice.moraes@univasf.edu.br

${ }^{4}$ Bioestatístico Embrapa Semiárido, Empresa Brasileira de Pesquisa Agropecuária (Embrapa), Rodovia BR-428 Km 152, Zona Rural, Petrolina, PE 56302-970, Brasil. E-mail: raimundo.parente@embrapa.br
}

Lactose-gema de ovo e resfriado a $5^{\circ} \mathrm{C} / 2 \mathrm{~h}$, envasado em palhetas de $0,5 \mathrm{~mL}$, e acondicionado sob vapor de nitrogênio líquido, e depois imersos. As amostras foram descongeladas $\left(37^{\circ} \mathrm{C} / 30 \mathrm{~s}\right)$ e avaliadas. As variáveis foram avaliadas com ANOVA e teste de Tukey $(\mathrm{P}<0,05)$. A motilidade total e progressiva foi maior $(\mathrm{P}<0,05)$ no sêmen tratado com CCC comparado as amostras do grupo controle, e CCC promoveu maior percentual $(\mathrm{P}<0,05)$ de motilidade total e progressiva durante as 3 horas de incubação. A percentagem de espermatozoides com viabilidade e integridade foi maior $(\mathrm{P}<0,05)$ no sêmen tratado com CCC $(81,47$ e $86,07 \%)$ comparado ao controle (72,12 e 70,19\%). 0 número de espermatozoides reativos ao teste hiposmótico foi maior $(\mathrm{P}<0,05)$ nas amostras de sêmen tratadas com CCC comparado ao controle. Adição de colesterol no sêmen de garanhões Nordestino melhora a qualidade espermática apos a criopreservação.

TERMOS DE INDEXAÇÃO: Colesterol, ciclodextrina, criopreservação, espermatozoides, garanhões, equinos, Equus caballus, membrana plasmática, motilidade, longevidade, viabilidade. 


\section{INTRODUÇÃo}

A raça Nordestina é descendente do cavalo Barbo-árabe, originário de Portugal e Espanha e introduzido no Brasil no período colonial, ao qual se assemelha morfologicamente (Costa et al. 1974). Esta raça se caracteriza pela rusticidade, sobriedade, rigidez muscular, temperamento sociável e amistoso, fácil adaptação ao meio e ao processo de criação, sendo utilizada principalmente no Nordeste brasileiro para o trabalho com o gado, pois podem sobreviver com pouco alimento e água, viajando grandes distâncias (Santos et al. 2015). Entretanto, com o fechamento da Associação Brasileira dos Criadores do Cavalo Nordestino (ABCCN) no início da década de 90, muitos dos acasalamentos passaram da atividade submetida a controle para não controlada, sendo que fêmeas têm sido acasaladas com reprodutores Mangalarga Marchador e Quarto-de-Milha para produção de mestiços para vaquejadas e os melhores machos estão sendo castrados e utilizados no trabalho com o gado (Costa et al. 2001). Desse modo, é de fundamental importância o desenvolvimento de programas que promovam a seleção, preservação e reprodução desse genoma, principalmente, em virtude do risco de extinção da raça.

A espécie equina apresenta elevados índices de variabilidade na qualidade espermática dentro da própria espécie e entre outras espécies de animais domésticos. Sendo um dos motivos para ocorrência deste fato, o devido critério de seleção dos reprodutores, adotado pelos criadores e associações, os quais levam em consideração principalmente a genealogia e o desempenho atlético, deixando em segundo plano os parâmetros de fertilidade (Uçan et al. 2016). Dessa forma, é favorecida a ocorrência de variabilidade na resistência espermática entre garanhões frente aos processos da criopreservação (Aurich \& Aurich 2006).

Os espermatozoides de alguns equinos apresentam tamanha sensibilidade a baixas temperaturas, que somente é possível utilizar o sêmen no estado fresco, situação na qual são reduzidos os benefícios gerados pela inseminação artificial para um considerável número de reprodutores (Aurich 2008). Essa sensibilidade é devido o espermatozoide de equinos possuir menor relação colesterol:fosfolipídios e maior relação proteínas:fosfolipídios, acarretando maior sensibilidade aos danos causados pelo resfriamento (Moraes et al. 2010). Estas características da membrana do espermatozoide equino aumentam a probabilidade de ocorrer perturbações resultantes do resfriamento e alteração nas interações entre os lipídios e proteínas, tornando-a mais sensível ao resfriamento que da espécie bovina (Moraes et al. 2010).

No entanto, pesquisas tem demonstrado que a incorporação do colesterol na membrana espermática, protege a célula espermática durante o resfriamento, além de reduzir os danos celulares decorrentes da criopreservação (Moraes et al. 2015, Souza et al. 2016a). Adicionalmente, o colesterol também promove à estabilização e modulação da fluidez da membrana a temperatura corpórea (Lee et al. 2015, Yildiz et al. 2015).

Portanto, na busca por melhores resultados na conservação da célula espermática na espécie equina, são necessários novos estudos sobre os vários aspectos relacionados à fisiologia da célula espermática quando submetida à refrigeração, congelação e descongelação. Diante disso, este estudo teve como objetivo avaliar o efeito da adição do colesterol carregado com ciclodextrina sobre a viabilidade e integridade da membrana plasmática de espermatozoides de garanhões Nordestino.

\section{MATERIAL E MÉTODOS}

Ética. 0 presente estudo foi realizado após a aprovação institucional da Universidade Federal do Vale do São Francisco (Univasf), sob o protocolo no 0006/161012 estando de acordo com os princípios éticos de experimentação animal do Comitê de Ética e Deontologia em Estudos e Pesquisas da Univasf.

Local de execução. 0 experimento foi realizado no Centro de Pesquisa em Suínos, Espécies Nativas e Silvestre (CPSENS), localizados no Campus de Ciências Agrárias da Univasf, situados no munícipio de Petrolina-PE (latitude $09^{\circ} 23^{\prime} 55^{\prime \prime}$ Sul e a uma longitude $40^{\circ} 30^{\prime} 03^{\prime \prime}$ Oeste) estando a uma altitude de 376 metros com precipitação média anual em torno de $300 \mathrm{~mm}$. A região apresenta temperatura média anual em torno dos $27^{\circ} \mathrm{C}$ e clima do tipo Bsh segundo a classificação de Peel et al. (2007).

Preparo do colesterol. 0 colesterol carregado com ciclodextrina foi preparado como descrito por Moraes et al. (2010). Brevemente, $200 \mathrm{mg}$ de colesterol foram dissolvidos em $1 \mathrm{~mL}$ de clorofórmio e $1 \mathrm{~g}$ de metil- $\beta$-ciclodextrina dissolvido em $2 \mathrm{~mL}$ de metanol. Em seguida alíquota de $0,45 \mathrm{~mL}$ da solução de colesterol foi adicionada à solução de ciclodextrina e agitada até a combinação das soluções ficarem clara. A solução resultante foi colocada em placa de petri de vidro para remoção dos solventes, por meio do fluxo de gás de nitrogênio com pureza mínima de $90 \%$. Logo após os cristais resultantes foram colocados para secar em placa aquecedora por 24 horas, e decorrido este tempo foram estocados em tubos de vidro a $22^{\circ} \mathrm{C}$. A solução de trabalho de colesterol carregado com ciclodextrina (CCC) foi preparada pela adição de $50 \mathrm{mg}$ de CCC em $1 \mathrm{~mL}$ do TALP a $37^{\circ} \mathrm{C}$ e homogeneizada por agitação com auxílio do agitador de tubos.

Animais. Dois garanhões da raça Nordestina, com idade média de 9,5 anos foram selecionados através de exame andrológico, adotando-se como adequados os critérios estabelecidos pelo Colégio Brasileiro de Reprodução Animal (CBRA 2013). Antes do período experimental, os garanhões foram submetidos ao esgotamento das reservas espermáticas extragonadais realizando-se coletas diárias, por oito dias, utilizando-se vagina artificial modelo Hannover ${ }^{\circledR}$ (Biotech, Botucatu/SP, Brasil) e uma fêmea em estro natural ou induzido como manequim.

Os garanhões foram mantidos em baias individuais, sendo clinicamente sadios e provenientes da Fazenda da Associação Brasileira de Criadores do Cavalo Nordestino, localizada em Juazeiro, BA $\left(09^{\circ} 25^{\prime} 00^{\prime \prime}\right.$ de latitude $S ; 40^{\circ} 30^{\prime} 00^{\prime \prime}$ de longitude 0). No local, os garanhões recebiam água ad libitum e alimentação com dieta composta de ração industrializada (Durancho ${ }^{\circledast} 12 \mathrm{MA}$ ), além de capim bermuda vaqueiro (Cynodon dactylon) e capim cameron (Pennisetum purpureum) picados e sal mineral, de acordo com as exigências para animais desta categoria segundo o NRC (2007).

Coleta e processamento do sêmen. Após $72 \mathrm{~h}$ do esgotamento dos garanhões, iniciou-se a coleta do sêmen para análise, realizando-se duas coletas por semana para cada garanhão $(n=20)$.

Após cada coleta, o ejaculado foi filtrado com auxílio do filtro de nylon e o volume determinado por visualização direta em Tubo Falcon graduado. Em seguida efetuou-se a avaliação física do sêmen como, aspecto (aquoso, leitoso e cremoso), coloração (branca, branca-amarelada e amarelada), odor e, sob microscopia convencional, a motilidade individual progressiva (0-100\%) e vigor $(0-5)$, onde foram utilizados apenas ejaculados que atendiam aos padrões considerados pelo CBRA (2013).

A diluição foi efetuada com sêmen (1:1) com diluidor de transporte (BotuSêmen ${ }^{\circledR}$, Biotech Botucatu, Botucatu, SP, Brasil), e o ejaculado foi transportado em uma caixa térmica para o CPSENS, mantido em banho-maria a $35^{\circ} \mathrm{C}$, 
No CPSENS, a concentração espermática foi determinada utilizando um fotômetro (Spermacue ${ }^{\circledR}$, Minitub, Berlin, Alemanha). Para isto, utilizou-se uma cubeta contendo $3,0 \mathrm{~mL}$ de água destilada e $120 \mu \mathrm{L}$ do sêmen diluído. 0 sêmen foi homogeneizado e submetido à centrifugação em tubos cônicos ( $50 \mathrm{~mL})$ a $400 \mathrm{~g}$ por 7 minutos. Após a centrifugação, o sobrenadante foi descartado e os peletes resultantes da centrifugação foram ressuspendidos no diluidor de congelação (BotuCrio ${ }^{\circledR}$, Biotech Botucatu, Botucatu/SP, Brasil) pré-aquecido a $35^{\circ} \mathrm{C}$, para a concentração de $120 \times 10^{6} \mathrm{sptz} / \mathrm{mL}$ e avaliados quanto à motilidade total (MT) e progressiva (MP) utilizando o sistema de análise computadorizada (CASA ${ }^{\circledR}$, Minitub, Berlin, Alemanha), equipado com o SpermVision ${ }^{\circledR}$. Ejaculados com menos 70\% de MT foram descartados.

Para determinação dos tratamentos experimentais o CCC foi adicionado ao sêmen diluído, estabelecendo os tratamentos: controle (sem adição do CCC), 0,75mg de CCC e 1,0mg de CCC/120x10 $\mathrm{sptz} / \mathrm{mL}$. Logo após as amostras foram retiradas do banho maria e mantidas a $22^{\circ} \mathrm{C}$, por $15 \mathrm{~min}$, para iniciar a curva de congelação. Após esse período, o sêmen foi diluído 1:5 (v/v) com diluente Lactose-Gema de ovo e acondicionado em câmara fria a $5{ }^{\circ} \mathrm{C}$ por duas horas. Depois, as amostras de cada tratamento foram envasadas em palhetas de $0,5 \mathrm{~mL}$ e lacradas com seladora (UltraSeal ${ }^{\circledR}$, Minitub, Berlim, Alemanha) e acondicionadas sob vapores do nitrogênio liquido, por 10 minutos $\left(-60^{\circ} \mathrm{C} / \mathrm{min}\right)$. Decorrido este tempo, as palhetas foram imersas no nitrogênio líquido $\left(-196^{\circ} \mathrm{C}\right)$ e estocadas em botijão criogênico para posterior análise.

Descongelação do sêmen. A descongelação foi realizada mergulhando-se as palhetas com as amostras de sêmen no descongelador automático (Cryofarm ${ }^{\circledR}$, IMV, São Paulo, São Paulo, Brasil) por 30 segundos a $37^{\circ} \mathrm{C}$.

Avaliação da motilidade espermática. Duas palhetas de cada tratamento foram descongeladas conforme descrito acima para avaliação da motilidade espermática (total e progressiva), utilizando o sistema de análise computadorizada (CASA). 0 conteúdo de cada palheta foi diluído 1:8 (v:v) com o diluente BotuSêmen ${ }^{\circledR}$, para concentração final de $50 \times 10^{6} \mathrm{sptz} / \mathrm{mL}$ e cada uma das amostras mantidas a temperatura ambiente por 5 min antes da serem analisadas (Spizziri et al. 2010). Alíquotas de $8 \mu \mathrm{L}$ de cada amostra por tratamento foram analisadas, individualmente, sobre lâminas pré-aquecidas, a $37^{\circ} \mathrm{C}$.

Avaliação da viabilidade espermática. Após a descongelação, o conteúdo de cada palheta foi transferido para microtubo de $2 \mathrm{~mL}$ contendo $1 \mathrm{~mL}$ do diluente Tris. Logo após, os espermatozoides foram corados para análise de fluorescência, transferindo $0,5 \mathrm{~mL}$ da amostra contida no microtubo para um segundo microtubo contendo $0,5 \mathrm{~mL}$ de Tris, $5 \mu \mathrm{L}$ SYBR-14 $\left(20 \mu \mathrm{M}\right.$ em $\left.\mathrm{Me}_{2} \mathrm{SO}\right)$ e $10 \mu \mathrm{L}$ de Iodeto de Propídio (IP; 2,4mM em água destilada; Molecular Probes, Eugene OR) como descrito por Santos et al. (2015).

As amostras foram incubadas por $10 \mathrm{~min}$ a $22^{\circ} \mathrm{C}$ antes de serem analisadas utilizando microscópio de fluorescência (AXIO Image $\mathrm{A2}{ }^{\circledR}$, Carl Zeiss, Berlim, Alemanha). Então, alíquotas de $10 \mu \mathrm{L}$ de cada amostra foram colocadas entre lâmina e lamínula pré-aquecidas a $37^{\circ} \mathrm{C}$ e um total de 200 células por amostra foram analisados. SYBR14 cora o DNA em verde na região nuclear dos espermatozoides, marcando as células vivas. 0 PI também cora DNA, porém, corando em vermelho as células com danos na membrana, logo, células inviáveis.

Avaliação da integridade da membrana plasmática. A integridade da membrana plasmática dos espermatozoides descongelados foi determinada pelo teste com sonda fluorescente. Para esta avaliação utilizou-se a associação de duas sondas fluorescentes, iodeto de propídio (IP) e Hoechst 33342 (H33342), conforme Souza et al. (2016b). As amostras de cada tratamento foram colocadas em microtubos de 1,5mL após descongelação. Então, alíquotas de $10 \mu \mathrm{L}$ de cada tratamento foram colocadas em outro microtubo, no qual foi adicionado $2 \mu \mathrm{L}$ PI e de $\mathrm{H} 33342$ e incubadas em banho maria a $37^{\circ} \mathrm{C}$ durante 8 minutos. Após este período, $10 \mu \mathrm{L}$ de cada amostra foram colocados entre lâmina e lamínula pré-aquecidas a $37^{\circ} \mathrm{C}$ e avaliadas em microscópio de fluorescência (AXIO Image $\mathrm{A} 2^{\circledR}$, Carl Zeiss, Berlin, Alemanha). Os espermatozoides com membrana plasmática lesada apresentavam o núcleo corado de rosa e os de membrana plasmática intacta possuem o núcleo corado de azul.

Avaliação da longevidade dos espermatozoides descongelados. Os espermatozoides descongelados foram submetidos ao teste de termo resistência (TTR) para avaliação da longevidade após descongelação. A avaliação consistiu no acondicionamento de $1,0 \mathrm{~mL}$ de cada amostra em microtubo de $1,5 \mathrm{~mL}$ em banho-maria a $37^{\circ} \mathrm{C}$ por 180 minutos. Onde $10 \mu \mathrm{L}$ de cada amostra no microtubos foram retiradas a cada 30 minutos $(0,30,60,90,120,150$ e $180 \mathrm{~min})$ para avaliação da motilidade total e progressiva no CASA (Souza \& Moraes 2017).

Avaliação da funcionalidade da membrana plasmática. Para avaliação da funcionalidade da membrana plasmática utilizou-se o teste hiposmótico (HOST) em câmara úmida, como descrito recentemente por Martins et al. (2011). Para tanto, amostras de $100 \mu \mathrm{L}$ foram adicionadas em microtubos de $1,5 \mathrm{~mL}$ e diluídas com $100 \mu \mathrm{L}$ da solução hiposmótica constituída por partes iguais de citrato tri-sódico e frutose obedecendo a concentração de $125 \mathrm{mOsm} / \mathrm{L}$. Logo após, as amostras diluídas foram incubadas em banho maria por $60 \mathrm{~min}$ a $37^{\circ} \mathrm{C}$. Transcorrido esse tempo às amostras foram formolizadas em solução de formol-salina tamponada na proporção de 10:1, sendo então estocadas em microtubo de 1,5mL para posterior avaliação.

Para avaliação foram depositados $10 \mu \mathrm{L}$ da solução incubada sobre lâmina, coberta com lamínula para proceder à contagem de $200 \mathrm{sptz} / \mathrm{lâmina} /$ tratamento, utilizando microscópio óptico com contraste de fase (DM 750 ${ }^{\circledR}$, Leica Microsystems, Heerbrugg, Suiça), em aumento de 400x.

Análise estatística. As variáveis foram submetidas à análise de variância e as médias comparadas pelo teste de Tukey a 5\% de probabilidade utilizando-se o programa SAS 9.2 (SAS 2009).

\section{RESULTADOS}

0 efeito do colesterol carreado com ciclodextrina sobre a motilidade espermática, viabilidade e integridade da membrana plasmática após a congelação/descongelação nos espermatozoides de garanhões são apresentados na Figura 1. A porcentagem de motilidade total e progressiva dos espermatozoides de garanhões foram maiores nas amostras tratadas com $0,75 \mathrm{mg}$ de CCC, quando comparado ao trato com 1,0mg de CCC e o grupo controle (Fig.1). Observa-se ainda que os espermatozoides tratados com $0,75 \mathrm{mg}$ de CCC, apresentaram maior percentual de viabilidade espermática e integridade de membrana plasmática em comparação aos demais tratamentos (Fig.1).

Os espermatozoides tratados com $0,75 \mathrm{mg}$ de colesterol carreado com ciclodextrina antes da criopreservação apresentaram maiores percentuais de motilidade total e progressiva durante as 3 horas de incubação, quando comparado aos demais tratamentos (Fig.2 e 3).

Em relação à funcionalidade da membrana plasmática, observa-se que o número de espermatozoides reativos ao HOST, após o processo de congelação/descongelação foi maior naqueles tratados com $0,75 \mathrm{mg}$ de CCC quando comparado ao grupo tratado com 1,0mg de CCC e o controle (Quadro 1). 
Quadro 1. Percentual espermático reativo ao teste hiposmótico de espermatozoides de garanhões da raça Nordestina tratados com colesterol carreado com ciclodextrina (CCC)

\begin{tabular}{|c|c|c|c|c|c|c|}
\hline & \multicolumn{6}{|c|}{ Reativos ao teste hiposmótico } \\
\hline & \multicolumn{3}{|c|}{$0 \mathrm{~m} 0 \mathrm{sm}$} & \multicolumn{3}{|c|}{$125 \mathrm{~m} 0 \mathrm{sm}$} \\
\hline & Controle & 0,75 CCC & $1,0 \mathrm{CCC}$ & Controle & 0,75 CCC & $1,0 \mathrm{CCC}$ \\
\hline Cauda reta & $110 \pm 3,3^{\mathrm{A}}$ & $93 \pm 3,2^{\mathrm{B}}$ & $99 \pm 3,1^{B}$ & $112 \pm 3,0^{\mathrm{A}}$ & $94 \pm 3,2^{\mathrm{B}}$ & $98 \pm 2,9^{\mathrm{B}}$ \\
\hline Cauda enrolada & $90 \pm 3,6^{\mathrm{B}}$ & $107 \pm 3,8^{\mathrm{A}}$ & $101 \pm 3,5^{\mathrm{A}}$ & $88 \pm 3,2^{\mathrm{B}}$ & $106 \pm 3,1^{\mathrm{A}}$ & $102 \pm 3,0^{\mathrm{A}}$ \\
\hline
\end{tabular}

$\overline{\mathrm{A}, \mathrm{B}}$ Médias \pm D.P seguidas de letras diferentes na mesma linha diferem entre si $(\mathrm{P}<0,05)$.

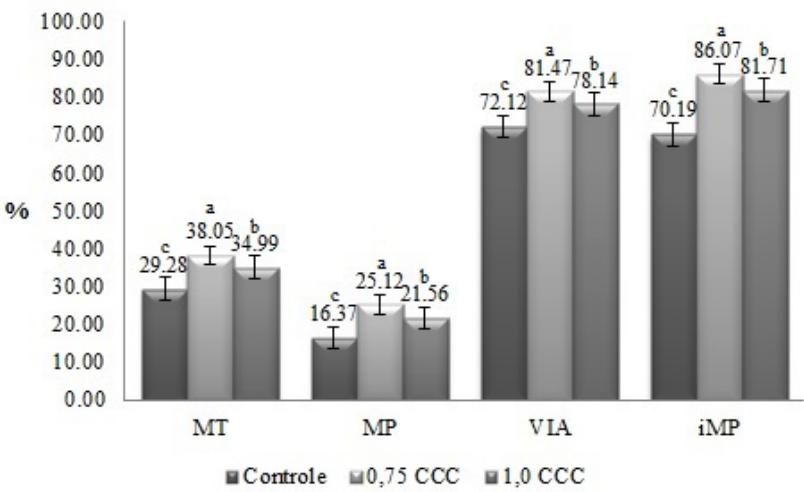

Fig.1. Motilidade total (MT) e progressiva (MP), viabilidade espermática e integridade de membrana plasmática de espermatozoides descongelados de garanhões Nordestino tratados com colesterol carreado com ciclodextrina (CCC); $\mathrm{P}<0,05$.

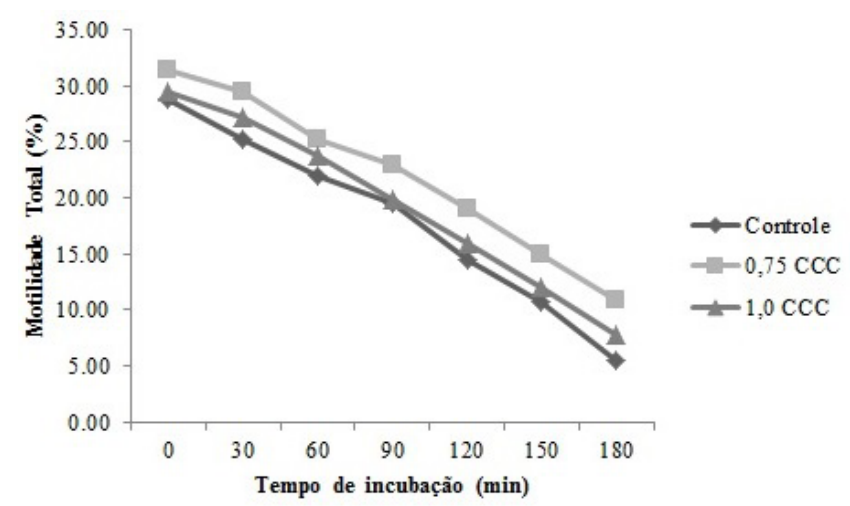

Fig.2. Motilidade total (\%) de espermatozoides de equinos até $3 \mathrm{~h}$ de incubação tratados com colesterol carreado com ciclodextrina (CCC); $\mathrm{P}<0,05$.

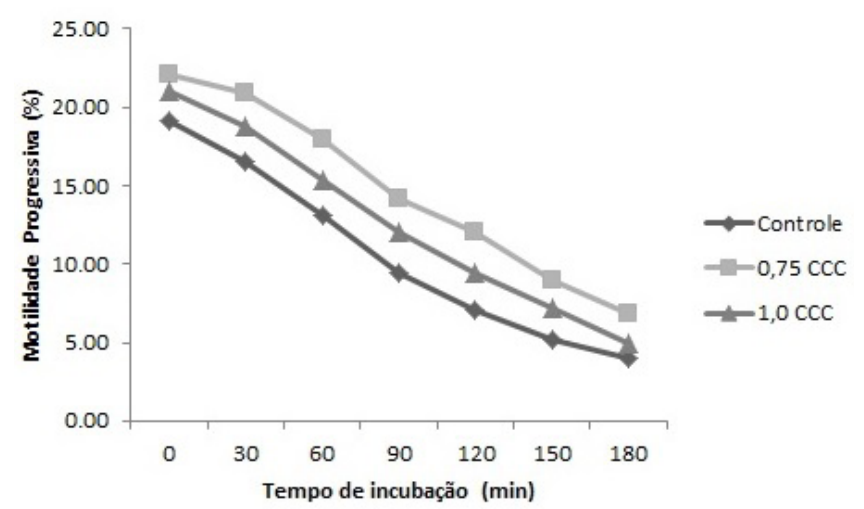

Fig.3. Motilidade progressiva (\%) de espermatozoides de equinos até $3 \mathrm{~h}$ de incubação tratados com colesterol carreado com ciclodextrina (CCC); $\mathrm{P}<0,05$.

\section{DISCUSSÃO}

A criopreservação dos espermatozoides de garanhões é uma biotecnologia que apesar das inúmeras vantagens, o processo de congelação/descongelação, na maioria das vezes, resulta em espermatozoides com pouca capacidade fecundante. Sendo a baixa relação colesterol:fosfolipídios presente na membrana espermática, uma das particularidades que propiciam uma sensibilidade na célula espermática, a baixas temperaturas. Essa sensibilidade favorece a formação de cristais de gelo intracelular, resultando em danos estruturais na membrana plasmática durante a fase de transição (Mocé \& Graham 2006). Além disso, as crioinjúrias favorecem a capacitação espermática precoce e reduzem a longevidade espermática, prejudicando diretamente a sua viabilidade e deslocamento no trato genital feminino (Uçan et al. 2016). Todavia, outros fatores podem influenciar no processo de capacitação espermática e a tolerância dos espermatozoides as baixas temperaturas, entretanto, o colesterol está envolvido diretamente em ambos os eventos (Moraes et al. 2010). Assim, nesse estudo buscamos minimizar a sensibilidade dos espermatozoides de garanhões da raça Nordestina, particularmente no que se refere à tolerância a baixas temperaturas, elevando o teor de colesterol na membrana espermática.

Este estudo confirma que o tratamento dos espermatozoides de garanhões com o colesterol carreado com ciclodextrina antes do processo de criopreservação, preservou a integridade da membrana plasmática, além de manter a viabilidade em níveis elevados após a congelação/descongelação, conforme achados anteriores (Mocé et al. 2010, Moraes et al. 2015, Souza et al. 2016a, Uçan et al. 2016), onde o tratamento dos espermatozoides com CCC proporcionou uma melhoria na fluidez da membrana plasmática em temperaturas reduzidas, favorecendo a resistência contra as crioinjúrias. Dessa forma, podemos considerar que, o tratamento utilizado $0,75 \mathrm{mg}$ de CCC, está dentro do nível de concentração adequado para a espécie, favorecendo a sua incorporação na membrana espermática, elevando assim a tolerância a lesões decorrentes da refrigeração e congelação/descongelação.

Nesse estudo podemos observar que o tratamento dos espermatozoides com o CCC manteve o percentual de motilidade espermática elevada ao longo das $3 \mathrm{~h}$ de incubação, sugerindo que essa manutenção da longevidade dos espermatozoides tratados com CCC venha favorecer a taxa de fecundidade de forma efetiva, uma vez que mantendo o percentual de motilidade por um maior período de tempo, é provável que os espermatozoides apresentem deslocamento no trato reprodutivo feminino até a fecundação do oócito (Souza et al. 2016a).

Apesar do percentual de motilidade apresentado pelos espermatozoides tratados com CCC, é possível observar uma 
redução dependente do tempo na motilidade espermática. Essa redução é esperada e pode ser atribuída ao declínio no metabolismo de energia dos espermatozoides que, ao serem expostos, por um longo período de incubação, acredita-se que ocorra um déficit de energia pela elevação do metabolismo celular e uma rápida utilização dos constituintes do diluente, implicando em danos diretos as organelas envolvidas na movimentação espermática, como a mitocôndria, prejudicando a atividade da cadeia de transporte de elétrons, consequentemente, provocando a redução na motilidade (Souza et al. 2016b). Contudo, os resultados apresentados revelaram o sêmen com valores de motilidade espermática para garanhões, dentro do percentual exigido pelo CBRA (2013), inferindo assim, a sua aptidão para fecundação.

A funcionalidade da membrana plasmática dos espermatozoides é fundamental para que ocorra a capacitação espermática e reação acrossomal (Siqueira et al. 2007). Assim, observou-se que a incorporação do CCC a membrana dos espermatozoides do cavalo Nordestino proporcionou uma manutenção da funcionalidade da membrana plasmática, conservando a sua integridade durante todo o processo de criopreservação. Essa preservação da funcionalidade da membrana plasmática obtida com a incorporação do colesterol é de suma importância, uma vez que em estudos realizados com jumentos (Canisso et al. 2008), Quarto de Milha (Costa et al. 2014) e Mangalarga Marchador (Costa et al. 2014, Pugliesi et al. 2012), o qual não adicionaram o CCC, apresentaram percentuais de funcionalidade da membrana plasmática inferiores.

Vale ressaltar que os resultados desse estudo mostraram que ao tratar os espermatozoides com CCC antes da criopreservação, elevou-se a relação colesterol:fosfolipídio, o que resultou em uma maior fluidez da membrana plasmática, favorecendo a redução do efeito deletério ocasionado pelas crioinjúrias na membrana espermática, durante os procedimentos de congelação/descongelação. Dessa forma, estudos adicionais são necessários no âmbito do tratamento com outras concentrações de CCC.

\section{CONCLUSÕES}

A adição do colesterol carreado com ciclodextrina ao sêmen diluído, antes da criopreservação, apresentou efeitos benéficos sobre a viabilidade e conservação da membrana plasmática dos espermatozoides de garanhões.

Os efeitos protetores da adição do colesterol carreado com ciclodextrina sobre a membrana dos espermatozoides de garanhões Nordestino sugerem que é possível aperfeiçoar a criopreservação do sêmen destes garanhões e preservação da raça através da inseminação artificial.

Agradecimentos. À FACEPE pelo financiamento a esta pesquisa (APQ-10725.04/12). À empresa BOTUPHARMA pela doação dos diluentes utilizados (BotuSêmen ${ }^{\circledR}$ e BotuCrio $^{\circledR}$ ). Ao CPSENS/FINEP por toda infraestrutura e suporte para a realização da pesquisa. À Associação Equestre e de Preservação do Cavalo Nordestino pela concessão do uso dos animais.

\section{REFERÊNCIAS}

Aurich C. 2008. Recent advances in cooled-semen technology. Anim. Reprod. Sci. 107(3/4):268-275. http://dx.doi.org/10.1016/j.anireprosci.2008.04.015. PMid:18524507.
Aurich J.E. \& Aurich C. 2006. Developments in european horse breeding and consequences for veterinarians in equine reproduction. Reprod. Domest. Anim. 41(4):275-279. http://dx.doi.org/10.1111/j.1439-0531.2006.00719.x. PMid:16869881.

Canisso I.F., Souza F.A., Ker P.G., Rodrigues A.L., Sena T.C. \& Carvalho G.R. 2008. Coleta de sêmen de jumentos (Equus asinus) utilizando-se éguas em estro como manequim. Ciênc. Vet. Tróp. 11(2/3):57-64.

CBRA 2013. Manual para Exame Andrológico e Avaliação de Sêmen Animal. 3a ed. Colégio Brasileiro de Reprodução Animal, Belo Horizonte, p.37.

Costa N., Lopes do Vale L.J. \& Leite G.U. 1974. Estudo da Preservação do Cavalo Nordestino. Departamento de Produção Animal, Recife, p.25.

Costa H.E., Filho H.C. \& Ferreira L. 2001. Exterior e Treinamento do Cavalo. Imprensa Universitária da Universidade Federal Rural de Pernambuco, Recife, p.51.

Costa D.N.M., Silva D.A.M., Boakari W.L., Ferreira S.B., Branco M.A.C. \& Souza J.A.T. 2014. Eficiência dos diluidores Tris e BotuCrio ${ }^{\circledR}$ sobre os parâmetros seminais de garanhões das raças Quarto de Milha e Mangalarga Marchador. Ciênc. Anim. Bras. 15(3):322-329. http://dx.doi.org/10.1590/1809$6891 v 15 i 325327$.

Lee Y.S., Lee S., Lee S.H., Yang B.K. \& Park C.K. 2015. Effect of cholesterolloaded-cyclodextrin on sperm viability and acrosome reaction in boar semen cryopreservation. Anim. Reprod. Sci. 159:124-130. http://dx.doi. org/10.1016/j.anireprosci.2015.06.006. PMid:26091957.

Martins L.F., Pinho R.O., Paraizo R.M., Oliveira R.R., Castilho E.F. \& Guimarães J.D. 2011. Avaliação de diferentes osmolaridades de soluções hiposmóticas e tempos de incubação no teste hiposmótico do sêmen de touros Nelore. Revta Bras. Zootec. 40(7):2019-2025. http://dx.doi.org/10.1590/S151635982011000700017 .

Mocé E. \& Graham J.K. 2006. Cholesterol-loaded cyclodextrins added to fresh bull ejaculates improve sperm cryosurvival. J. Anim. Sci. 84(4):826-833. http://dx.doi.org/10.2527/2006.844826x. PMid:16543559.

Mocé E., Blanch E., Tomás C. \& Graham J.K. 2010. Use of cholesterol in sperm cryopreservation: present moment and perspectives to future. Reprod. Domest. Anim. 45(Suppl.2):57-66. http://dx.doi.org/10.1111/j.14390531.2010.01635.x. PMid:20591066.

Moraes E.A., Graham J.K., Torres C.A.A., Meyers M. \& Spizziri B. 2010. Delivering cholesterol or cholestanol to bull sperm membranes improves cryosurvival. Anim. Reprod. Sci. 118(2/4):148-154. http://dx.doi.org/10.1016/j. anireprosci.2009.08.002. PMid:19733986.

Moraes E.A., Matos W.C.G., Graham J.K. \& Ferrari Junior W.D. 2015. Cholestanolloaded-cyclodextrin improve the quality of stallion spermatozoa after cryopreservation. Anim. Reprod. Sci. 158:19-24. http://dx.doi.org/10.1016/j. anireprosci.2015.04.004. PMid:26005214.

NRC 2007. Nutrient Requirements of Small Ruminants: Sheep, Goats, Cervids, and New World Camelids. National Requirement Council, National Academy of Science, Washintgton, D.C., p.245.

Peel M.C., Finlayson B.L. \& McMahon T.A. 2007. Updated world map of the Köppen-Geiger climate classification. Hydrol. Earth Syst. Sci. 11(5):16331644. http://dx.doi.org/10.5194/hess-11-1633-2007.

Pugliesi G., Fürst R. \& Carvalho G.R. 2012. Efeito de diferentes tempos de equilíbrio na criopreservação de sêmen de garanhões. Revta Bras. Ciênc. Vet. 19(3):172-177.

Santos M.A.M., Gradela A., Moraes E.A., Souza W.L., Alves N.G., Costa J.M.S. \& Matos W.C.G. 2015. Características do sêmen a fresco e descongelado de garanhões da raça Nordestina. Pesq. Vet. Bras. 35(11):925-932. http:// dx.doi.org/10.1590/S0100-736X2015001100009.

SAS 2009. Statistical Analysis System 2002-2008: System for Windows, Versão 9.2. SAS Institute Inc., Cary, USA.

Siqueira J.B., Guimarães J.D., Costa E.P., Henry M., Torres C.A.A., Silva M.V.G.B. \& Silveira T.S. 2007. Relação da taxa de gestação com sêmen bovino 
congelado e testes de avaliação espermática in vitro. Revta Bras. Zootec 36(2):387-395. http://dx.doi.org/10.1590/S1516-35982007000200016.

Souza W.L. \& Moraes E.A. 2017. Dimetilformamida adicionada no sêmen de caprinos e seu efeito sobre a longevidade e funcionalidade da membrana espermática após criopreservação. Revta Semiárido de Visu 5:11-20.

Souza W.L., Moraes E.A., Costa J.M.S. \& Graham J.K. 2016a. Cholesterol-loaded cyclodextrin in fresh goat sperm improves cryosurvival rates. Revta Bras. Ciênc. Vet. 23(1/2):93-98.

Souza W.L., Moraes E.A., Costa J.M.S., Sousa P.H.F., Lopes Junior E.S., Oliveira R.P. \& Toniolli R. 2016b. Efeito de diferentes concentrações de melatonina em espermatozoides de carneiros sobre estresse oxidativo após criopreservação. Pesq. Vet. Bras. 36(7):657-664. http://dx.doi. org/10.1590/S0100-736X2016000700017.
Spizziri B.E., Fox M.H., Bruemmer J.E., Squires E.L. \& Graham J.K. 2010. Cholesterol-loaded-cyclodextrins and fertility potential of stallions spermatozoa. Anim. Reprod. Sci. 118(2/4):255-264. http://dx.doi. org/10.1016/j.anireprosci.2009.08.001. PMid:19762177.

Uçan U., Küçük N., Ahmad E., Naseer Z., Aksoy M., Serin İ. \& Ceylan A. 2016. Effect of different sugars supplemented to the extender in combination with cholesterol-loaded cyclodextrin (CLC) on post-thaw quality of ram spermatozoa. Small Rumin. Res. 136:243-246. http://dx.doi.org/10.1016/j. smallrumres.2016.01.021.

Yildiz C., Yavas I., Bozkurt Y. \& Aksoy M. 2015. Effect of cholesterol-loaded cyclodextrin on cryosurvival and fertility of cryopreserved carp (Cyprinus carpio) sperm. Cryobiology 70(2):190-194. http://dx.doi.org/10.1016/j. cryobiol.2015.01.009. PMid:25661710. 